Original Contribution

\title{
ACUTE FLACCID PARALYSIS REGISTERED FOR THE PERIOD 2012-2020, IN STARA ZAGORA REGION
}

\author{
I. Simeonova ${ }^{1}$, Sv. Petrova ${ }^{2}$, I. Mladenova ${ }^{1^{*}}$ \\ ${ }^{1}$ Department of Hygiene, Epidemiology, Microbiology, Parasitology and Infectious Diseases, \\ Faculty of Medicine, Trakia University, Stara Zagora, Bulgaria \\ ${ }^{2}$ Regional Health Inspectorate, Stara Zagora, Bulgaria
}

\begin{abstract}
Acute Flaccid Paralysis (AFP) is a clinical syndrome. There are many infectious and non-infectious causes of AFP. Poliomyelitis caused by the wild polio virus (the natural circulating strain of polio) is one of the causes of AFP. As a part of the worldwide campaign to eradicate polio, all countries do surveillance for polio by looking for clinical cases of AFP. The purpose of the study is to describe the cases of Acute Flaccid Paralysis, without Paresis Nervi facialis, in the Stara Zagora region for the period 2012-2020. Methods: A retrospective, descriptive analysis was performed on the parameters: diagnosis, seasonality, sex, residence, age, clinical data, and comorbidities, results of follow-up examinations, polio vaccine administration, and timeliness of studies. Results: There were 9 cases of AFP, without Paresis nervi facialis, registered and reported for the Stara Zagora region, for the period $2012-2020$. The children in 3 years old were more affected- 4 patients. About the coverage with polio vaccine: 7 of the patients had 4 doses, 1 - had 6 doses, 1 child had 5 doses, and +1 zero dose. 100\% of children are covered, according to their age, with IPV. Follow-up in 6 of the patients is without residual paresis and complications. Residual paresis was found in 1 of the cases. In 2 patients no control examination was performed due to migration. Conclusion: The correct epidemiological diagnosis is the way to timely and corrects clinical diagnosis. AFP Surveillance is of particular importance as well as fecal sampling up to 48 hours, from the onset of paralysis, and follow-up after the 60th day in children $<15$ years.
\end{abstract}

Key words: Acute Flaccid Paralysis (AFP), polio eradication, inactivated polio vaccine (IPV)

\section{INTRODUCTION}

Enterovirus infections are acute infectious diseases with different clinical features. They are transmitted by fecal-oral and airborne routes of transmission, through food, water and contaminated hands. Distribution is ubiquitous. In the 70 s of the 20th century, so called "new enteroviruses" were discovered, with neurological symptoms- types 70 and 71 . In 1975 in Bulgaria an epidemic of enterovirus 71 was registered with a high case-fatality rate, especially in young children. (1) In bulbar forms respiratory and cardiac paralysis occurs. During that epidemic, 89 paralytic cases with $29 \%$ case fatality rate in ages 1-3 years were observed. (2) Enteroviruses belong to the

\footnotetext{
*Correspondence to Irena Mladenova, Department of Hygiene, Epidemiology and Infectious Diseases, Faculty of Medicine, Trakia University, 6000 Stara Zagora, Bulgaria. E-mail: imladenova@yahoo.com; tel: 08973244 72
}

genus Enterovirus (EV) from the familyPicornaviridae. They were classified into twelve species according to the International Committee on the Taxonomy of Viruses (ICTV) (3).

Acute Flaccid Paralysis (AFP) is a clinical syndrome. There are many infectious and noninfectious causes of AFP. Poliomyelitis caused by the wild polio virus (the natural circulating strain of polio) is one of the causes of AFP. As a part of the worldwide campaign to eradicate polio, all countries do surveillance for polio by looking for clinical cases of AFP. The clinical syndrome of AFP is defined as the acute onset of weakness or paralysis, with reduced muscles tone in children $<15$ years (4). As an indicator of the country's ability not to miss polio, serves the detection, even in the absence of polio, of at least one case of AFP per 100,000 children < 15 years. 
SIMEONOVA I., et al.

The wild poliovirus 2 was eradicated in 2015 . In 2019, the World Health Organization (WHO) announced the eradication of wild poliovirus 3 . Wild poliovirus 1 continues to circulate. In 1988, the World Health Assembly set WHO goal of achieving global polio eradication by 2000 . In the year the initiative was launched, there were more than 350,000 new cases of paralytic polio worldwide, in a total of 125 countries. In 2018 the incidence has decreased to 33 cases, in the region of Afghanistan and Pakistan (poliovirus type 1), and the next 2019 the cases were 71 . For the global polio eradication more than 2.5 billion children have been vaccinated, focusing on Afghanistan, Nigeria and Pakistan, due to the endemic nature of polio. The eradication has not been achieved in 2000 and hopes are postponed to $2023(5,6)$.

\section{PURPOSE AND TASKS}

The purpose of the study is to describe the cases of Acute Flaccid Paralysis (AFP) without Paresis nervi facialis, in the Stara Zagora region for the period 2012-2020. The data are retrospective on the occurred AFP in children $<15$ years registered in the Information System for Surveillance of AFP, at NCIPD, Sofia (7). The aim is to detect suspected or probable cases of polio. Certification of a country as polio-free territory requires that no new cases of poliomyelitis caused by wild poliovirus have been registered and reported.

\section{MATERIALS AND METHODS}

A retrospective, descriptive analysis was performed on the parameters: diagnosis, seasonality, sex, residence of living, age, clinical data, comorbidities, results of followup examinations, polio vaccine administration, and timeliness of epidemiological investigations and collection of fecal samples.

The data from the maps in the Information System for Surveillance of AFP in Bulgaria and attached medical documentation were used - history of disease, control examinations performed after 60 days from the onset of paralysis, telephone survey with GPs and follow-up specialist. Due to the delicacy of the problem and the residual paresis, as a severe physical condition, we rely on the anamnesis taken at the time of suffering, and filling in the questionnaire, and the retrospective study, incl. in active supervision.

\section{RESULTS}

There are 9 cases of AFP, without Paresis nervi facialis, registered and reported for Stara Zagora region, for the period 2012 - 2020 (Tables 1, 2).

Table1. Registered diagnoses

\begin{tabular}{|c|c|}
5 cases & Guillain-Barré syndrome \\
\hline 1 case & Encephalomyelopolyradiculoneuritis \\
\hline 1 case & Meningopolyradiculoneuritis \\
\hline 1 case & Damage to the lumbosacral plexus \\
\hline 1 case & Damage to the nervus fibularis sinistra \\
\hline
\end{tabular}

Table 2. Distribution by the year of occurrence

\begin{tabular}{|c|c|}
\hline 2012 & Guillain-Barré syndrome \\
\hline 2014 & Guillain-Barré syndrome \\
\hline 2015 & Encephalomyelopolyradiculoneuritis \\
\hline 2015 & Meningopolyradiculoneuritis \\
\hline 2016 & $\begin{array}{c}\text { Damage to the lumbosacral plexus } \\
\text { Damage to the nervus fibularis sinistra }\end{array}$ \\
\hline 2018 & Guillain-Barré syndrome \\
\hline 2019 & Guillain-Barré syndrome \\
\hline 2020 & Guillain-Barré syndrome \\
\hline
\end{tabular}




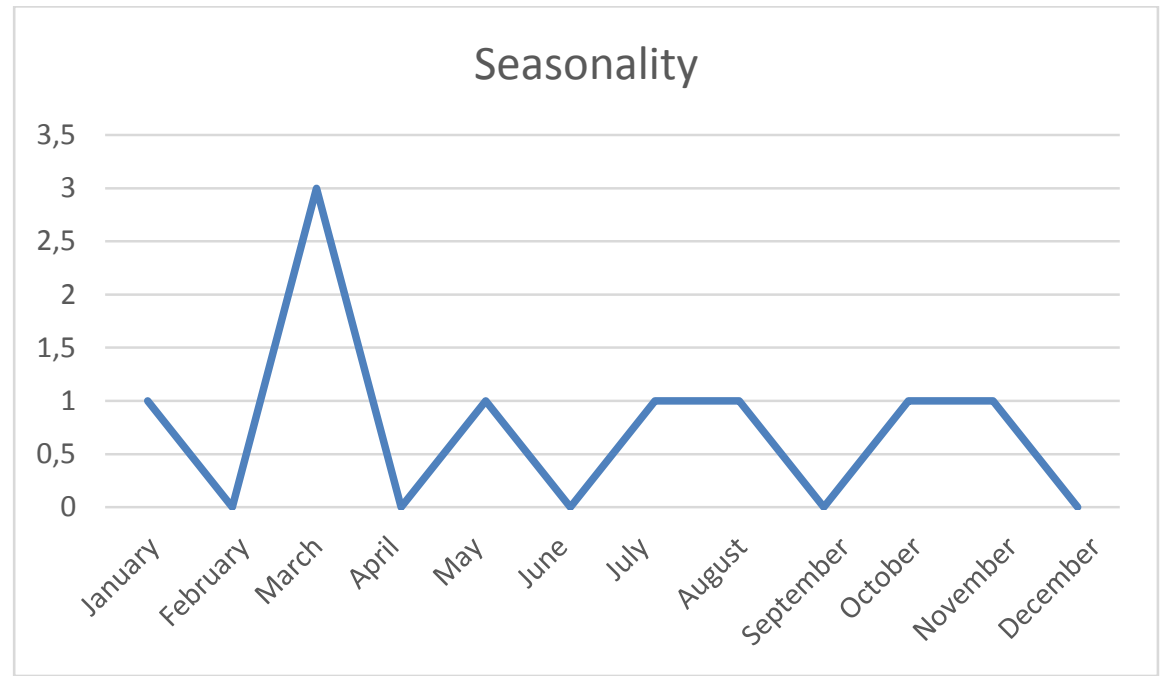

Figure 1. Distribution of patients by month.

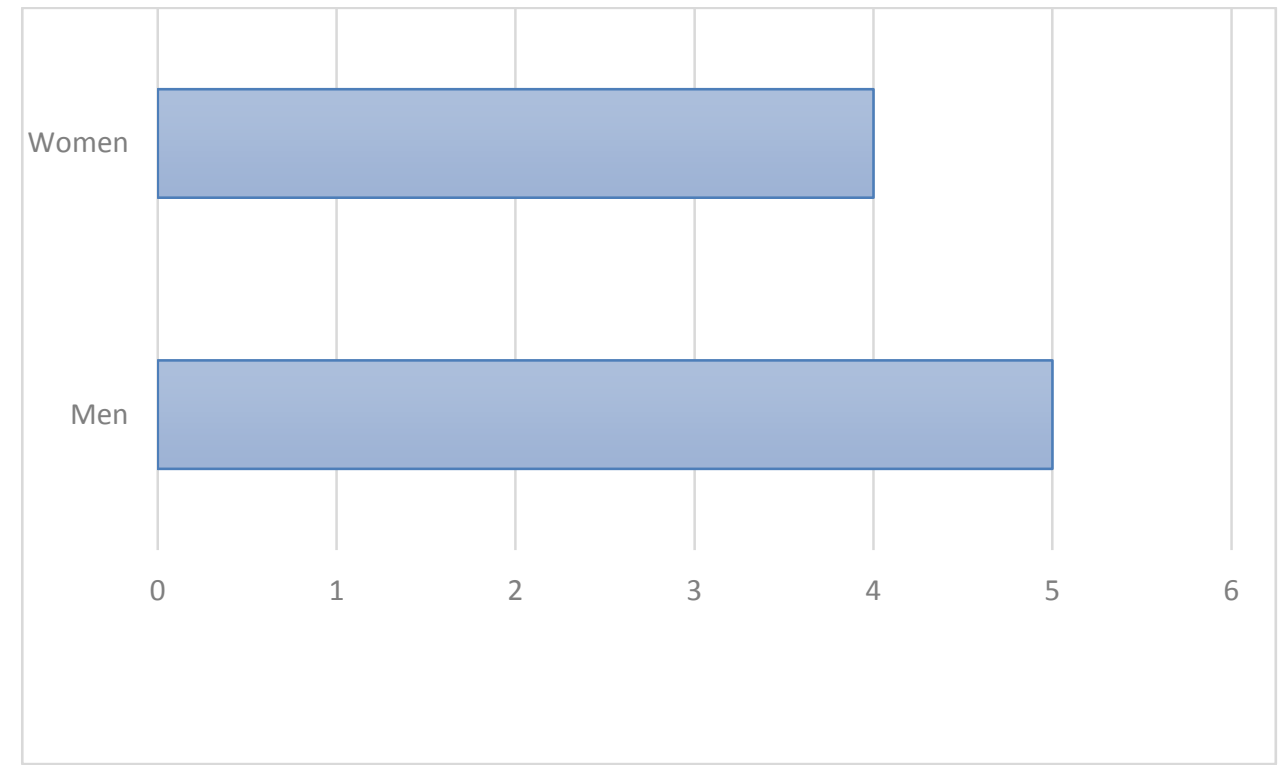

Figure 2. Gender distribution of patients.

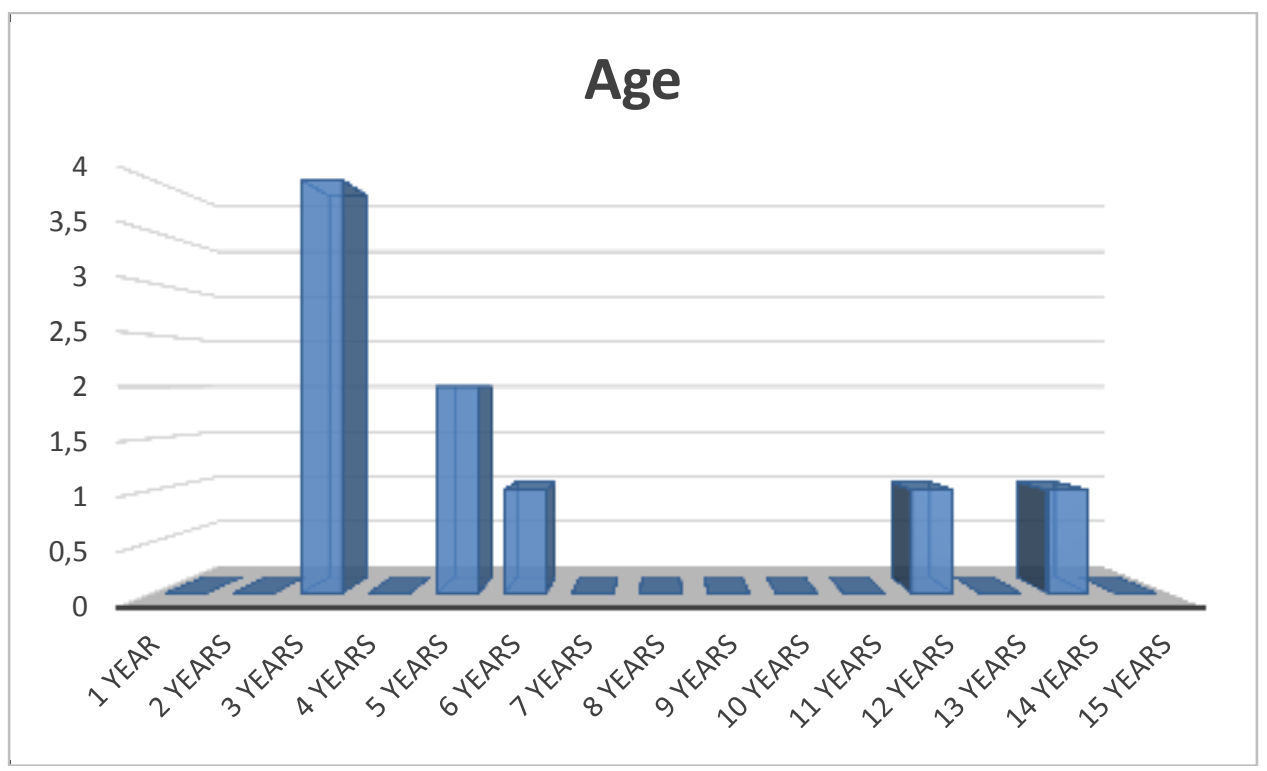

Figure 3. Age distribution of patients. 
There was not an observed seasonality (yearround) (Figure 1). The distribution by sex was as follows - 5 men and 4 women (Figure 2). The children in 3 years old are more affected4 patients (Figure 3). No ethnic or racial characteristics were identified. The distribution by place of residence was: town-7, village- 2 cases. About the coverage with polio vaccine: 7 of the patients had 4 doses, 1 - had 6 doses, 1 child had 5 doses and +1 zero dose. $100 \%$ of children were covered, according to their age, with IPV.

Timely epidemiological investigations were performed in 7 of the patients. In the remaining 2 cases due to the active detection and bringing from another ward and diagnosis AFP, in the differential diagnostic plan, the epidemiological studies were later. Two fecal samples were taken on two consecutive days, within 48 hours of the onset of paralysis. Contact children $<15$ years of age, in the respective household, were studied during the primary epidemiological investigation, as well as their immunization status with regard to the polio vaccine.

The disease begins as AVI (acute viral infection) in 1 child with enteric syndrome; in 5 other children - it has a temperature $\geq 37.2$ $38.5^{\circ} \mathrm{C}$. Pain and stiffness of the affected limbs were observed in 5 children; watery diarrhea and repeated vomiting - 1 case; double vision 1. In one child, on the 3rd day after immunization with IPV (inactivated polio vaccine) there were complaints of headache, and the 20th day- complaints of weakness of the legs and "double" vision begin.

Concomitant diseases were: bronchitis - 1 child, epilepsy - 1 , thalassemia minor -1 , and AVI - 1 child. Relatives of 1 child were diagnosed with lupus erythematosus.

All patients were treated in a hospital (6 patients- in "University Hospital St. Georgi", in Plovdiv, 2 children- in the University Hospital "Prof. Dr. Stoyan Kirkovich", in Stara Zagora, and 1 child - in "Prof. Ivan Mitev", Sofia. Diagnosis was confirmed in the reference laboratory "Enteroviruses", in NCIPD, Sofia, without a proven cause such as poliovirus. Only in one case, enterovirus was detected by molecular diagnostics (PCR).
Control examinations were held after the 60th day. Follow-up in 6 of the patients is without residual paresis and complications. Residual paresis was found in 1 of the cases. In 2 patients no control examination was performed due to migration. One child was diagnosed with epilepsy, and one child- with a weakened achilles reflex.

\section{CONCLUSIONS}

The correct and targeted epidemiological diagnosis is a way to timely and corrects clinical diagnosis. AFP Surveillance is of particular importance (timely reporting) as well as fecal sampling up to 48 hours, from the onset of paralysis, of AFP cases, and follow-up after the 60th day in children $<15$ years. There was high immunization coverage with polio vaccine, and the immunization status was complete, for the respective age.

\section{REFERENCES}

1. Ribarova N., Stoilova Y., Valkanova N., Mladenova - Hristova I., Mitova - Mineva Y. Epidemiology of Infectious diseases. Simelpress, pp 447, 2011.

2. Kaneva J. Handbook of Infectious Diseases. Siela, pp 224, 1999.

3. Xiaobo Lei, Xia Xiao and Jianwei Wang. Innate Immunity Evasion by Enteroviruses: Insights

4. into Virus-Host Interaction. Viruses, 8, 22, 2016; doi:10.3390/v8010022

5. National Institute for Communicable Diseases. Outbreak Response Unit, \& Centre for Vaccines and Immunology. Polio eradication and acute flaccid paralysis (AFP) surveillance. Frequently Asked Questions. January 2018, https://www.nicd.ac.za/

6. Nikolaeva - Glomb L., Stoyanova A., Georgieva I. Polyomyelitis. Is eradication close? Medinfo 2020, (1). https://www.medinfo.bg/spisanie/2020/1/st atii/poliomielit-blizo-li-e-eradikacijata3078

7. https://polioeradication.org/poliotoday/history-of-polio/. Посетено на 25.02.2021г.

8. https://afp.gateway.bg/ WEB BASED SYSTEM FOR SURVEILLANCE OF ACUTE FLACCID PARALYSIS (AFP) IN BULGARIA 